\title{
Parameter Estimation Model of Weibull Distribution on Students' Achievement of Mathematic Education Program, Cenderawasih University
}

\author{
Yan Dirk Wabiser \\ Lecturer, Pancasila and Civic \\ Education Dept. of Uncen \\ Cenderawasih University \\ .Abepura Street, Sentani
}

\author{
Mayor M. H. Manurung \\ Lecturer, Mathematics education \\ Dept. of Uncen \\ Cenderawasih University \\ Abepura Street, Sentani
}

\author{
Halomoan Edy Manurung \\ Lecturer, Information System Dept. \\ of UOGP \\ Ottow Geissler University, Papua \\ Perkutut Street, Kotaraja Dalam
}

\begin{abstract}
This research aims to analyze and implement a distribution model obtained from grade result data of students of Mathematic Education Program so as to present the most useful information while their achievements are one factor of education quality control. For these reasons, this research investigates students' achievement. The data are gained from their results of final semester exams on several subjects for the past three years. The analysis is conducted by clustering the subjects completed by the students. One of continual distribution used is Weibull distribution. The distribution attained is estimated by the Maximum Likelihood Estimation (MLE) method using Newton Raphson iteration. This research is supposed to deepen the statistical concept and numerical method particularly Weibull distribution using Maximum Likelihood Estimation along with Newton Raphson iteration and their application as well as to provide the Study program of Mathematic Education with problem solving alternatives and information to enhance learning quality in order to control the progress of students' achievement on subjects learned and motivate them. (learning subjects).
\end{abstract}

\section{Keywords}

Parameter Estimation Model, Weibull Distribution, Maximum Likelihood Estimation Method, Subject Results/Grades

\section{INTRODUCTION}

\subsection{Background}

One of the current government programs is to improve the quality of education nationally. An increase in quality at school level is designed to provide public community with education services. For this reason, the government has been trying to offer opportunity to citizens to get education by sufficient facilities supplied. In relation to the current development of the country, the government attempts to adjust the educational systems to the needs of all field development that require expertise and skills to enlarge productivity, creativity and work efficiency.

However, people assume that one's success is determined by his or her highest formal education in educational system of Indonesia. As a consequence, a lot of high school graduates apply to attend at universities annually either public or private universities. This is due to the fact that the number of students exceeds the capacity. The candidates must compete each other in doing entrance test. The test is tight. If they pass, they will be able to attend the program chosen. In facts, some of accepted students fail to complete their education within the period of time designed by the program and even they fail at all at the beginning. The success in learning is measured by grade results of tests then they are quantified in grade average point (GPA). If we want to identify students' learning process, we can access from their results every semester accomplished because their results reflect their learning process occurred [5].

Bear in mind that learning achievement is one factor of education quality control, the writers conducted this research at Study Program of Mathematic Education. The analysis was done by clustering all completing subjects. The distribution used was one of continual distribution i.e. Weilbull Distribution. The obtained distribution is estimated by the Maximum Likelihood Estimation (MLE) method with Newton Raphson iteration.

\subsection{Problem Formulation}

Referring to the background, the problems of this research are formulated as follows:

a. How is parameter estimation obtained using Weibull distribution?

b. How do distribution model interpret data of grades/results obtained from the subjects accomplished at Study Program of Mathematic Education, Cenderawasih University?

\subsection{Previous Research}

There have been numerous studies conducted related to the use of Weibull Distribution method. Firstly, a research entitles function of national exam result prediction in Papua Province with Weibull distribution approach [6]. This research explains that the result of Weibull distribution can be used to predict the results of national exam of the following year based on the results and conditions of the previous year. The prediction result shows that the results of national exams in Papua province are much better than other coastal and mountainous areas. Jayapura municipality represents the province to gain the excellent grades in some subjects namely Chemistry, Physics, and Math. Biak district represents coastal areas to have an excellent grade in Biology subject.

Another research conducted in Nabire entitles Comparison of Parameter Estimation Methods to Determine the Frequency Data Magnitude of Aftershock in Nabire, Papua[7]. This research explicates that the results of comparison of three methods to estimate weibull distribution parameter. The methods used are maximum likelihood estimation, mean square error, and graphs obtained from magnitude frequency data of aftershocks in Nabire district. These methods were examined by the Kolmogrov-Smirnov to identify their normality distribution. The result shows that the best method used is maximum likelihood estimation. 
In addition, a research under the title of the Analysis of Phytoplankton Abundance Using Weibull Distribution (A Case Study in the Csoastal Area of East Yapen in the Regency of Yapen Islands, Papua) [8] uses Weibull distribution method. This study elucidates the use of weibull distribution parameter with maximum likelihood estimation method in order to identify the abundance of phytoplankton on 15 water sites along the coast of Yapen Timur. The parameter shape is about 3,9844 and the parameter scale is 79,929 . The highest composition result of phytoplankton abundance is Bacillariophyceae (50\%) and the least is Phyrrophyceae (9\%) and Cyanophceae.

\subsection{Aims and Contributions}

The followings are significance and contribution of the research:

a. Researchers : they can implement the concept of inferential statistics - Weibull Distribution - in their related research.

b. Mathematic Education Science: it can be used to enrich the insights of science and its application toward parameter estimation model especially weibull distribution.

c. Education: it can give problem solving alternatives and information to improve the learning quality in education and view the progress of achievement as well as learning motivation.

\section{THEORY, MODEL AND ANALYSIS}

\subsection{Mechanism and Procedure of Assessment}

In accordance to the manual of assessment issued by the Standardized Board of National Education (Badan Nasional Standar Pendidikan (BNSP)), it highlights some principles that should be paid in attention on assessment procedure as follows:

a. The assessment is designed to measure competency achievement. For that reason, it should be understood that the assessment is a part of teaching and learning activities done by a teacher in order to know the achievement level of graduate competency standard;

b. The assessment uses criteria reference, a decision is taken based on students' results after the learning process. This should be built upon the competence based curriculum and criterion reference to compare students' results achieved;

c. The assessment is done totally and continually. The assessment is prepared as a part of learning process and the process of assessment can be done on time range of learning process. If a student successfully achieves the standard, he or she passes, he or she, however, fails to achieve the standard result; he or she must take a remedial class to achieve a minimum results of the standard required.

d. The results of assessment determine the follow-up action; the actions can be a revision of learning process, a remedial program for students whose grades are under the minimum standard and an enrichment program for students whose grades reach the standard of completeness criteria.

e. The assessment must be appropriate with students' learning experience during learning process. This is related closely to the comprehension on an integrated assessment of total learning process. This is referred to the government regulation of PP No. 19 year 2005 regarding the assessment of educational process in which it is categorized into three parts namely learning assessments conducted by a teacher, by school and by the government.

\subsection{Two-parameter Weibull distribution}

Weibull distribution is one of the first continuous distribution esthablished by Swedish physicist named Waloddi Weibull in 1939. Weibull distribution density function can be expressed as:

$$
\begin{gathered}
f(t, \alpha, \beta)= \\
\frac{\beta}{\alpha}\left(\frac{t}{\alpha}\right)^{\beta-1} \exp \left[-\left(\frac{t}{\alpha}\right)\right]^{\beta} .
\end{gathered}
$$

Weibull cumulative distribution function is:

$$
F(t, \alpha, \beta)=1-\exp \left[-\left(\frac{t}{\alpha}\right)\right]^{\beta}
$$

So that the survival function is:

$$
S(t, \alpha, \beta)=1-\left\{1-\exp \left[-\left(\frac{t}{\alpha}\right)\right]^{\beta}\right\}
$$

This is t random variable, $\alpha$ is the scale parameter and $\beta$ is a shape parameter. Parameters $\alpha$ and $\beta$ are parameters to be estimated[4].

\subsection{Methods of Maximum Likelihood Estimations}

MLE method is one of parameter estimation methods that can be used to evaluate a model parameter of the already known distribution. To obtain a model of parameter estimation via MLE method is to maximize the function of likelihood.

Given that $X_{1}, X_{2}, \ldots$, are samples of $n$ sized from a population that its distribution depends on a set of parameters $p$, that $\theta 1, \theta 2, \ldots, \theta$ p. We can assume that this samples form a random vector $X=\left(X_{1}, X_{2}, \ldots, X_{n}\right)^{\prime}$. For example $\mathrm{X}$ has a density function $f(x, \theta)$, where $x=\left(x_{1}, x_{2}, \ldots, x_{n}\right)^{\prime}$ and $\theta=$ $\left(\theta_{1}, \theta_{2}, \ldots, \theta_{p}\right)^{\prime}$. This density function is normally called as the $\mathrm{X}$ likelihood function, symbolized by $L(x, \theta)$. Given sample of MLE from $\theta$, symbolized by $\hat{\theta}$, ), is the value of $\theta$ that maximizes $L(x, \theta)$.If $L(x, \theta)$ has a partial derivative towards $\theta 1$ $, \theta 2, \ldots, \theta \mathrm{p}$, so $\hat{\theta}$ can be obtained by solving equations:

$$
. . \frac{\partial L(x, \widehat{\theta})}{\partial \theta_{i}}=0, i=1,2, \ldots, p .
$$

In all situations, it is easy to do with logarithm $L(x, \theta)$, in which maxima achieved are on similar points with $L(x, \theta)$. So $\hat{\theta}$ fulfills the following equation::

$\frac{\partial \log [L(x, \widehat{\theta})]}{\partial \theta_{i}}=0, i=1,2, \ldots, p$.

Equation of (2.3.2) is known as likelihood equation. For instance $\mathrm{X} 1, \mathrm{X} 2, \ldots \mathrm{Xn}$ shape the $\mathrm{n}$ sized sample from normal distribution without knowing the mean $\mu$ and the variants $\sigma^{2}$.

Where $\theta=\left(\mu, \sigma^{2}\right)$, given the likelihood function is as follows:

$$
L(x, \theta)=\frac{1}{\left(2 \pi \sigma^{2}\right)^{\frac{n}{2}}} \exp \left[-\frac{1}{2 \sigma^{2}} \sum_{i=1}^{n}\left(x_{i}-\mu\right)^{2}\right]
$$

Given $L^{*}(x, \theta)=\log L(x, \theta)$ as a result:

$$
L^{*}(x, \theta)=-\frac{1}{2 \sigma^{2}} \sum_{i=1}^{n}\left(x_{i}-\mu\right)^{2}-\frac{n}{2} \log \left(2 \pi \sigma^{2}\right)
$$


Likelihood equation (2.3.2) is a configuration of :

$\frac{\partial L^{*}}{\partial_{\mu}}=\frac{1}{\sigma^{2}} \sum_{i=1}^{n}\left(x_{i}-\hat{\mu}\right)=0$.

$\frac{\partial L^{*}}{\partial \sigma^{2}}=\frac{1}{2 \widehat{\sigma}^{2}} \sum_{i=1}^{n}\left(x_{i}-\hat{\mu}\right)^{2}-\frac{n}{2 \widehat{\sigma}^{2}}=0$

Equation (2.3.2.1) and (2.3.2.2) can be written as follows:

$\mathrm{n}\left(\mathrm{x}^{-}-\mu^{\wedge}\right)=0 n(\bar{x}-\hat{\mu})=0$,.

$\sum_{i=1}^{n}\left(x_{i}-\hat{\mu}\right)^{2}-n \hat{\sigma}^{2}=0$.

Where $\bar{x}=(1 / n) \sum_{i=1}^{n} x_{i}$, if $n \geq 2$, as a consequence the equation of (2.3.2.3) and (2.3.2.4) has the following solution:

$$
\begin{gathered}
\hat{\mu}=\bar{x} \\
\widehat{\sigma}^{2}=\frac{1}{n} \sum_{i=1}^{n}\left(x_{i}-\bar{x}\right)^{2}
\end{gathered}
$$

These are the MLE from each $\mu$ and $\sigma^{2}$. This can be verified that $\hat{\mu}$ and $\hat{\sigma}^{2}$ are values of $\mu$ and $\sigma^{2}$ that maximalized $L^{*}(x, \theta)$ To show these, we look at the following Hessian matrix of the second order of $L^{*}$ partial derivative:

$$
A=\left[\begin{array}{cc}
\frac{\partial^{2} L^{*}}{\partial \mu^{2}} & \frac{\partial^{2} L^{*}}{\partial \mu \partial \sigma^{2}} \\
\frac{\partial^{2} L^{*}}{\partial \mu \partial \sigma^{2}} & \frac{\partial^{2} L^{*}}{\partial \sigma^{4}}
\end{array}\right]
$$

So for $\mu=\hat{\mu}$ and $\sigma^{2}=\hat{\sigma}^{2}$,

$$
\begin{gathered}
\frac{\partial^{2} L^{*}}{\partial \mu^{2}}=-\frac{n}{\widehat{\sigma}^{2}}, \\
\frac{\partial^{2} L^{*}}{\partial \mu \partial \sigma^{2}}=-\frac{1}{\widehat{\sigma}^{4}} \sum_{i=1}^{n}\left(x_{i}-\hat{\mu}\right)=0, \\
\frac{\partial^{2} L^{*}}{\partial \sigma^{4}}=-\frac{n}{2 \hat{\sigma}^{4}}
\end{gathered}
$$

Thus $\partial^{2} L^{*} / \partial \mu^{2}<0$ and $\operatorname{det}(A)=n^{2} / 2 \hat{\sigma}^{6}>0$. As a result $\left(\hat{\mu}, \hat{\sigma}^{2}\right)$ is the local maximum point of $L^{*}[1]$.

\subsection{Newton-Raphson Method}

Given $\mathrm{x}_{0}$ is the initial point of $f(x)$. Applying Taylor $\mathrm{f}$ expansion in $\mathrm{x}_{0}$ area enables to estimate $f(x)$ with square function $\phi(x)$ therefore:

$$
\phi(x)=f\left(x_{0}\right)+\left(x-x_{0}\right)^{\prime} \Delta f\left(x_{0}\right)+\frac{1}{2 !}\left(x-x_{0}\right)^{\prime} H_{f}\left(x_{0}\right)(x-
$$

Where $H_{f}\left(x_{0}\right)$ ) is $\mathrm{f}$ Hessian matrix that is evaluated in $x_{0}$. Based on the formula of (2.4.1) we can attain a worthy estimation of $f(x)$ minimal by using $\phi(x)$ minimal. If $\phi(x)$ reaches a local minimum at $x_{1}$, so we surely assure to have $\nabla \emptyset\left(x_{1}\right)=0$ as follows:

$$
\nabla f\left(x_{0}\right)+H_{f}\left(x_{0}\right)\left(x_{1}-x_{0}\right) . .
$$

If $H_{f}\left(x_{0}\right)$ is nonsingular, so the equation (2.4.2) can be gained:

$$
x_{1}=x_{0}-H_{f}^{-1}\left(x_{0}\right) \nabla f\left(x_{0}\right)
$$

If it is estimated $f(x)$ with other square functions, and reapplication of Taylor expansion in $x_{1}$ area and then repetition of similar process occurs as before with $x_{1}$ is used to replace $x_{0}$, the point obtained is as follows:

$$
x_{2}=x_{1}-H_{f}^{-1}\left(x_{1}\right) \nabla f\left(x_{1}\right)
$$

Further repetition of this process directs towards the point orders, $x_{0}, x_{1}, x_{2}, \ldots, x_{i}, \ldots$, thus the formula is as follows:

$x_{i+1}=x_{i}-H_{f}^{-1}\left(x_{i}\right) \nabla f\left(x_{i}\right), \quad i=0,1,2, \ldots \ldots$

Newton-Raphson method requires inverts from Hessian matrix $H_{f}$ at each iteration. Furthermore, this method fails if $\left.H_{f}\left(x_{i}\right)\right)$ is not positive definitif. This can occur, for example $x_{i}$ is far away from the location of $x^{*}$ minimum, the initial point $x_{0}$ however, is close to $x^{*}$ thus convergence will occur rapidly. NewtonRaphson method will be used for iteration so as to get converging estimator value[1]

\subsection{Analysis Research Methods}

\subsubsection{Data Source and Research Variables}

Data were collected from students' grade results of subjects from 2017 up to 2019 of Mathematic Education, Cenderawasih University. The software used to proceed the data is Maple 2016 and Matlab 2013. The research variable is Response variable, the average grades of subjects are categorized in each subject, they then are analyzed theoretically to obtain a parameter so as to analyze or to characterize the total data [2].

\subsubsection{Analysis Methods}

The analysis methods used are as follows:

1. To determine the Maximum Likelihood of data distribution with the following steps:

a) Survival function formed then it is derivative as follows:

$$
S(\text { Survival })=e^{-\left(\frac{t_{1, k}}{\alpha}\right)^{\beta}}
$$

b) Weibull distribution obtained from PDF (Probability Density Function) as follows:

$$
P(p d \text { furvival })=-\frac{\left(\frac{t_{1, k}}{\alpha}\right)^{\beta} \beta e^{-\left(\frac{t_{1, k}}{\alpha}\right)^{\beta}}}{t_{1, k}}
$$

c) Likelihood function formed as follows:

$$
L(\text { likelihoodsurvival })=\prod_{k}^{n} P_{k}(p d f \text { survival })
$$

d) Log transformation conducted on likelihood function as follows:

$$
L L(\log \text { likelihood })=\log \prod_{k=1}^{n} p d f_{k}=\sum_{k=1}^{n} \log p d f_{k}
$$

e) To maximize log function (formula/symbol) with partial derivative towards estimated parameters.

2. To study parameter estimations by using Newton-Raphson with the following stages:

a) Seeking gradient vectors that contain the following parameters:

$$
\vec{g}=\left(\begin{array}{l}
\frac{\partial L}{\partial \alpha} \\
\frac{\partial L}{\partial \beta}
\end{array}\right)
$$

b) Searching for Hessian matrix as follows: 


$$
\left(\begin{array}{ll}
\frac{\partial^{2} L}{\partial \alpha^{2}} & \frac{\partial^{2} L}{\partial \alpha \partial \beta} \\
\frac{\partial^{2} L}{\partial \beta^{2}} & \frac{\partial^{2} L}{\partial \beta \partial \alpha}
\end{array}\right)
$$

c) Writing the formula of Newton-Raphson as follows:

$$
\theta=\theta_{0}-H^{-1} g
$$

d) Algorithm creating in programming Newton Rapshon as follows:

$$
\hat{\theta}=\theta-\left.H^{-1} g\right|_{\theta}
$$

e) Iterasi Iteration is ended if ;

$$
\|\hat{\theta}-\theta\|<\varepsilon
$$

Where $\varepsilon$ the real number is too small, for example $\varepsilon=0.0005$.

f) The conclusion is thus estimator of MLE or distribution function as follows:

$$
\widehat{\theta}=\theta
$$

g) Estimator is attained from:

$$
\hat{\theta}=\theta^{n}
$$

3. After getting the estimator, the model is derived from factors containing in the average results of national exam.

4. To examine the hypothesis.

\begin{tabular}{|c|c|c|c|c|c|c|}
\hline \multicolumn{7}{|c|}{ INTEGRAL CALCULUS } \\
\hline Year & $\begin{array}{c}\text { Shape } \\
\text { Param } \\
\text { eter }\end{array}$ & $\begin{array}{c}\text { Scala } \\
\text { Param } \\
\text { eter }\end{array}$ & Mean & $\begin{array}{c}\text { Standa } \\
\text { r } \\
\text { Deviat } \\
\text { ion }\end{array}$ & $\begin{array}{l}\text { The } \\
\text { Peak } \\
\text { value }\end{array}$ & $\begin{array}{l}\text { Surv } \\
\text { ival } \\
\text { (70) }\end{array}$ \\
\hline 2017 & $\begin{array}{c}73.03 \\
22\end{array}$ & $\begin{array}{c}75.36 \\
38\end{array}$ & 76 & $\begin{array}{c}14.78 \\
02\end{array}$ & 0.356 & $98 \%$ \\
\hline 2018 & $\begin{array}{c}51.96 \\
86\end{array}$ & $\begin{array}{c}56.22 \\
43\end{array}$ & $\begin{array}{c}56.60 \\
00\end{array}$ & $\begin{array}{c}11.83 \\
92\end{array}$ & 0.339 & $0 \%$ \\
\hline
\end{tabular}

5. To interpret a model and take a conclusion [9]

\section{RESULTS AND DISCUSSION}

Table 1. Differential Calculus

\begin{tabular}{|c|c|c|c|c|c|c|}
\hline \multicolumn{7}{|c|}{ DIFFERENTIAL CALCULUS } \\
\hline Year & $\begin{array}{c}\text { Shape } \\
\text { Param } \\
\text { eter }\end{array}$ & $\begin{array}{c}\text { Scala } \\
\text { Param } \\
\text { eter }\end{array}$ & Mean & $\begin{array}{c}\text { Stand } \\
\text { ar } \\
\text { Deviat } \\
\text { ion }\end{array}$ & $\begin{array}{c}\text { The } \\
\text { Peak } \\
\text { value }\end{array}$ & $\begin{array}{c}\text { Surv } \\
\text { ival } \\
(60)\end{array}$ \\
\hline 2017 & 77.26 & 78.78 & 59.64 & 20.20 & 0.360 & $96 \%$ \\
& 43 & 64 & 00 & 29 & & \\
\hline 2018 & 49.57 & 48.00 & 49.52 & 14.12 & 0.380 & $0 \%$ \\
& 93 & 64 & 00 & 78 & & \\
\hline 2019 & 48.00 & 49.12 & 51.46 & 15.39 & 0.359 & $0 \%$ \\
& 87 & 54 & 54 & 53 & & \\
\hline
\end{tabular}

Table 2. Integral Calculus
Table 3. Algebra and Trigonometry

\begin{tabular}{|c|c|c|c|c|c|c|}
\hline \multicolumn{7}{|c|}{ ALGEBRA And TRIGONOMETRY } \\
\hline Year & $\begin{array}{c}\text { Shape } \\
\text { Param } \\
\text { eter }\end{array}$ & $\begin{array}{c}\text { Scala } \\
\text { Param } \\
\text { eter }\end{array}$ & Mean & $\begin{array}{c}\text { Stand } \\
\text { ar } \\
\text { Deviat } \\
\text { ion }\end{array}$ & $\begin{array}{c}\text { The } \\
\text { Peak } \\
\text { value }\end{array}$ & $\begin{array}{c}\text { Surv } \\
\text { ival } \\
(70)\end{array}$ \\
\hline 2017 & $\begin{array}{c}44.31 \\
11\end{array}$ & $\begin{array}{c}49.45 \\
54\end{array}$ & $\begin{array}{c}50.16 \\
00\end{array}$ & $\begin{array}{c}17.41 \\
33\end{array}$ & 0.329 & $0 \%$ \\
\hline 2018 & 70.63 & 74.66 & 75.12 & 7.933 & 0.347 & $98 \%$ \\
& 04 & 45 & 00 & 7 & & \\
\hline
\end{tabular}

Table 4. Field Geometrics

\begin{tabular}{|c|c|c|c|c|c|c|}
\hline \multicolumn{7}{|c|}{ FIELD GEOMETRICS } \\
\hline Year & $\begin{array}{c}\text { Shape } \\
\text { Param } \\
\text { eter }\end{array}$ & $\begin{array}{c}\text { Scala } \\
\text { Param } \\
\text { eter }\end{array}$ & Mean & $\begin{array}{c}\text { Stand } \\
\text { ar } \\
\text { Deviat } \\
\text { ion }\end{array}$ & $\begin{array}{c}\text { The } \\
\text { Peak } \\
\text { value }\end{array}$ & $\begin{array}{c}\text { Surv } \\
\text { ival } \\
(70)\end{array}$ \\
\hline 2017 & 66.80 & 69.57 & 70.40 & 22.36 & O.353 & $19 \%$ \\
& 70 & 57 & 00 & 44 & & \\
\hline 2018 & 54.62 & 55.68 & 58.52 & 18.44 & 0.360 & $0 \%$ \\
& 35 & 64 & 00 & 84 & & \\
\hline
\end{tabular}

Table 5. Spatial Geometrics

\begin{tabular}{|c|c|c|c|c|c|c|}
\hline \multicolumn{7}{|c|}{ SPATIAL GEOMETRICS } \\
\hline Year & $\begin{array}{c}\text { Shape } \\
\text { Para } \\
\text { meter }\end{array}$ & $\begin{array}{c}\text { Scala } \\
\text { Para } \\
\text { meter }\end{array}$ & Mean & $\begin{array}{c}\text { Stand } \\
\text { ar } \\
\text { Devia } \\
\text { tion }\end{array}$ & $\begin{array}{c}\text { The } \\
\text { Peak } \\
\text { value }\end{array}$ & $\begin{array}{c}\text { Sur } \\
\text { viv } \\
\text { al } \\
(70)\end{array}$ \\
\hline 2017 & 66.03 & 73.96 & 76.68 & 11.13 & 0.327 & 96 \\
& 25 & 54 & 00 & 82 & & $\%$ \\
\hline 2018 & 74.12 & 82.03 & 84.33 & 3.082 & 0.331 & 99 \\
& 24 & 41 & 33 & 2 & & $\%$ \\
\hline
\end{tabular}

Table 6. Basic Statistics

\begin{tabular}{|c|c|c|c|c|c|c|}
\hline \multicolumn{7}{|c|}{ BASIC STATISTICS } \\
\hline Year & $\begin{array}{c}\text { Shape } \\
\text { Para } \\
\text { meter }\end{array}$ & $\begin{array}{c}\text { Scala } \\
\text { Para } \\
\text { meter }\end{array}$ & Mean & $\begin{array}{c}\text { Stand } \\
\text { ar } \\
\text { Devia } \\
\text { tion }\end{array}$ & $\begin{array}{c}\text { The } \\
\text { Peak } \\
\text { value }\end{array}$ & $\begin{array}{c}\text { Sur } \\
\text { viva } \\
1 \\
(60)\end{array}$ \\
\hline 2017 & 58.57 & 62.67 & 65.60 & 12.12 & 0.343 & 92 \\
& 56 & 82 & 00 & 44 & & $\%$ \\
\hline 2018 & 62.88 & 64.02 & 63.48 & 73 & 0.361 & 97 \\
& 91 & 42 & 00 & & & \\
\hline 2019 & 57.02 & 66.62 & 68.80 & 12.44 & 0.313 & 99 \\
& 46 & 29 & 77 & 19 & & $\%$ \\
\hline
\end{tabular}


Table 7. Math Statistics I

\begin{tabular}{|c|c|c|c|c|c|c|}
\hline \multicolumn{7}{|c|}{ MATH STATISTICS I } \\
\hline Year & $\begin{array}{c}\text { Shape } \\
\text { Para } \\
\text { meter }\end{array}$ & $\begin{array}{c}\text { Scala } \\
\text { Para } \\
\text { meter }\end{array}$ & Mean & $\begin{array}{c}\text { Stand } \\
\text { ar } \\
\text { Devia } \\
\text { tion }\end{array}$ & $\begin{array}{c}\text { The } \\
\text { Peak } \\
\text { value }\end{array}$ & $\begin{array}{c}\text { Sur } \\
\text { viv } \\
\text { al } \\
(70)\end{array}$ \\
\hline 2017 & 79.00 & 80.54 & 79.05 & 19.04 & 0.360 & 99 \\
& 33 & 48 & 00 & 70 & & $\%$ \\
\hline 2018 & 75.96 & 76.04 & 78.54 & 17.67 & 0.367 & 99 \\
& 75 & 33 & 74 & 42 & & $\%$ \\
\hline 2019 & 77.03 & 81.06 & 80.28 & 9.085 & 0.349 & 99 \\
& 42 & 54 & 00 & 3 & & $\%$ \\
\hline
\end{tabular}

Table 8. Real Analysis I

\begin{tabular}{|c|c|c|c|c|c|c|}
\hline \multicolumn{7}{|c|}{ REAL ANALYSIS I } \\
\hline Year & $\begin{array}{c}\text { Shape } \\
\text { Para } \\
\text { meter }\end{array}$ & $\begin{array}{c}\text { Scala } \\
\text { Para } \\
\text { meter }\end{array}$ & Mean & $\begin{array}{c}\text { Stand } \\
\text { ar } \\
\text { Devia } \\
\text { tion }\end{array}$ & $\begin{array}{c}\text { The } \\
\text { Peak } \\
\text { value }\end{array}$ & $\begin{array}{c}\text { Sur } \\
\text { viva } \\
1 \\
(70)\end{array}$ \\
\hline 2017 & 75.23 & 78.74 & 80.73 & 9.347 & 0.351 & 99 \\
& 55 & 52 & 91 & 9 & 5 & $\%$ \\
\hline 2018 & 71.34 & 69.54 & 69.82 & 16.78 & 0.377 & 17 \\
& 66 & 69 & 61 & 92 & & $\%$ \\
\hline 2019 & 70.24 & 74.08 & 75.41 & 8.010 & 0.348 & 97 \\
& 36 & 96 & 67 & 4 & & $\%$ \\
\hline
\end{tabular}

Table 9. Real Analysis II

\begin{tabular}{|c|c|c|c|c|c|c|}
\hline \multicolumn{7}{|c|}{ REAL ANALYSIS II } \\
\hline Year & $\begin{array}{c}\text { Shape } \\
\text { Param } \\
\text { eter }\end{array}$ & $\begin{array}{c}\text { Scala } \\
\text { Param } \\
\text { eter }\end{array}$ & Mean & $\begin{array}{c}\text { Stand } \\
\text { ar } \\
\text { Devia } \\
\text { tion }\end{array}$ & $\begin{array}{c}\text { The } \\
\text { Peak } \\
\text { value }\end{array}$ & $\begin{array}{c}\text { Sur } \\
\text { viva } \\
1 \\
(70)\end{array}$ \\
\hline 2017 & 62.22 & 71.75 & 70.12 & 7.790 & 0.319 & $79 \%$ \\
08 & 70 & 00 & 6 & & \\
\hline 2018 & 71.63 & 76.68 & 76.72 & 6.318 & 0.343 & $99 \%$ \\
& 04 & 34 & 73 & 4 & & \\
\hline
\end{tabular}

Table 10. Introduction to Basic Math

\begin{tabular}{|c|c|c|c|c|c|c|}
\hline \multicolumn{7}{|c|}{ INTRODUCTION TO BASIC MATH } \\
\hline Year & $\begin{array}{c}\text { Shape } \\
\text { Param } \\
\text { eter }\end{array}$ & $\begin{array}{c}\text { Scala } \\
\text { Param } \\
\text { eter }\end{array}$ & Mean & $\begin{array}{c}\text { Standa } \\
\text { r } \\
\text { Deviat } \\
\text { ion }\end{array}$ & $\begin{array}{c}\text { The } \\
\text { Peak } \\
\text { value }\end{array}$ & $\begin{array}{c}\text { Sur } \\
\text { viva } \\
1 \\
(60)\end{array}$ \\
\hline 2017 & 69.00 & 72.00 & 71.80 & 14.80 & 0.352 & $99 \%$ \\
& 13 & 21 & 00 & 43 & & \\
\hline 2018 & 59.94 & 66.66 & 67.52 & 14.66 & 0.330 & $99 \%$ \\
& 52 & 25 & 00 & 32 & & \\
\hline
\end{tabular}

Table 11. Evaluation of Math Learning Achievement

\begin{tabular}{|c|c|c|c|c|c|c|}
\hline \multicolumn{6}{|c|}{ EVALUATION OF MATH LEARNING ACHIEVEMENT } \\
\hline Year & $\begin{array}{c}\text { Shape } \\
\text { Param } \\
\text { eter }\end{array}$ & $\begin{array}{c}\text { Scala } \\
\text { Param } \\
\text { eter }\end{array}$ & Mean & $\begin{array}{c}\text { Standa } \\
\text { r } \\
\text { Deviat } \\
\text { ion }\end{array}$ & $\begin{array}{c}\text { The } \\
\text { Peak } \\
\text { value }\end{array}$ & $\begin{array}{c}\text { Surv } \\
\text { ival } \\
(70)\end{array}$ \\
\hline 2017 & $\begin{array}{c}69.001 \\
3\end{array}$ & $\begin{array}{c}72.002 \\
1\end{array}$ & $\begin{array}{c}87.105 \\
3\end{array}$ & $\begin{array}{c}11.779 \\
9\end{array}$ & 0.352 & $81 \%$ \\
\hline
\end{tabular}

Table 12. Linear Algebra

\begin{tabular}{|c|c|c|c|c|c|c|}
\hline \multicolumn{7}{|c|}{ LINEAR ALGEBRA } \\
\hline Year & $\begin{array}{c}\text { Shape } \\
\text { Param } \\
\text { eter }\end{array}$ & $\begin{array}{c}\text { Scala } \\
\text { Param } \\
\text { eter }\end{array}$ & Mean & $\begin{array}{c}\text { Stand } \\
\text { ar } \\
\text { Deviat } \\
\text { ion }\end{array}$ & $\begin{array}{c}\text { The } \\
\text { Peak } \\
\text { value }\end{array}$ & $\begin{array}{c}\text { Sur } \\
\text { viva } \\
1 \\
(70)\end{array}$ \\
\hline 2017 & 79.05 & 78.06 & 80.16 & 3.975 & 0.372 & 99 \\
86 & 96 & 00 & 8 & & $\%$ \\
\hline 2018 & 71.85 & 75.00 & 74.88 & 7.759 & 0.352 & $98 \%$ \\
& 61 & 05 & 00 & & & \\
\hline 2019 & 81.05 & 82.04 & 83.12 & 9.510 & 0.363 & $99 \%$ \\
& 51 & 6 & 00 & 2 & & \\
\hline
\end{tabular}

Table 13. Research on Math Teaching

\begin{tabular}{|c|c|c|c|c|c|c|}
\hline \multicolumn{7}{|c|}{ RESEARCH ON MATH TEACHING } \\
\hline Year & $\begin{array}{c}\text { Shape } \\
\text { Param } \\
\text { eter }\end{array}$ & $\begin{array}{c}\text { Scala } \\
\text { Param } \\
\text { eter }\end{array}$ & Mean & $\begin{array}{c}\text { Standa } \\
\text { r } \\
\text { Deviati } \\
\text { on }\end{array}$ & $\begin{array}{c}\text { The } \\
\text { Peak } \\
\text { value }\end{array}$ & $\begin{array}{c}\text { Surv } \\
\text { ival } \\
(70)\end{array}$ \\
\hline 2017 & $\begin{array}{c}73.343 \\
6\end{array}$ & $\begin{array}{c}68.061 \\
2\end{array}$ & $\begin{array}{c}75.181 \\
8\end{array}$ & 3.2898 & 0.394 & $2 \%$ \\
\hline 2018 & 72.850 & 74.068 & 5 & 74.480 \\
& 1 & 5 & 5 & 12.718 & 0.361 & $96 \%$ \\
& & & 0 & & \\
\hline 2019 & 75.022 & 81.689 & 83.291 & 9.6255 & 0.337 & $99 \%$ \\
& 6 & 9 & 7 & & & \\
\hline
\end{tabular}

Table 14. Number Theory

\begin{tabular}{|c|c|c|c|c|c|c|}
\hline \multicolumn{7}{|c|}{ NUMBER THEORY } \\
\hline Year & $\begin{array}{c}\text { Shape } \\
\text { Param } \\
\text { eter }\end{array}$ & $\begin{array}{c}\text { Scala } \\
\text { Param } \\
\text { eter }\end{array}$ & Mean & $\begin{array}{c}\text { Standa } \\
\text { r } \\
\text { Deviat } \\
\text { ion }\end{array}$ & $\begin{array}{c}\text { The } \\
\text { Peak } \\
\text { value }\end{array}$ & $\begin{array}{c}\text { Surviva } \\
1(70)\end{array}$ \\
\hline 2017 & $\begin{array}{c}62.452 \\
1\end{array}$ & $\begin{array}{c}64.674 \\
3\end{array}$ & $\begin{array}{c}63.560 \\
0\end{array}$ & $\begin{array}{c}12.887 \\
6\end{array}$ & 0.355 & $0 \%$ \\
\hline 2018 & 69.560 & 71.041 & 71.600 & 12.506 & 0.359 & $67 \%$ \\
& 1 & 1 & 0 & & & \\
\hline 2019 & 70.020 & 71.191 & 70.120 & 13.248 & 0.361 & $71 \%$ \\
& 3 & 0 & 0 & 6 & & \\
\hline
\end{tabular}


Table 15. Linear Program

\begin{tabular}{|c|c|c|c|c|c|c|}
\hline \multicolumn{7}{|c|}{ LINEAR PROGRAM } \\
\hline Year & $\begin{array}{c}\text { Shape } \\
\text { Param } \\
\text { eter }\end{array}$ & $\begin{array}{c}\text { Scala } \\
\text { Param } \\
\text { eter }\end{array}$ & Mean & $\begin{array}{c}\text { Standa } \\
\mathrm{r} \\
\text { Deviat } \\
\text { ion }\end{array}$ & $\begin{array}{c}\text { The } \\
\text { Peak } \\
\text { value }\end{array}$ & $\begin{array}{c}\text { Surviva } \\
1(60)\end{array}$ \\
\hline 2018 & $\begin{array}{c}63.211 \\
5\end{array}$ & $\begin{array}{c}67.021 \\
5\end{array}$ & $\begin{array}{c}66.280 \\
0\end{array}$ & $\begin{array}{c}22.552 \\
8\end{array}$ & 0.346 & $99 \%$ \\
\hline
\end{tabular}

Table 16. Differential Equation

\begin{tabular}{|c|c|c|c|c|c|c|}
\hline \multicolumn{7}{|c|}{ DIFFERENTIAL EQUATION } \\
\hline Year & $\begin{array}{c}\text { Shape } \\
\text { Param } \\
\text { eter }\end{array}$ & $\begin{array}{c}\text { Scala } \\
\text { Param } \\
\text { eter }\end{array}$ & Mean & $\begin{array}{c}\text { Standa } \\
\text { r } \\
\text { Deviati } \\
\text { on }\end{array}$ & $\begin{array}{c}\text { The } \\
\text { Peak } \\
\text { value }\end{array}$ & $\begin{array}{c}\text { Surv } \\
\text { ival } \\
(70)\end{array}$ \\
\hline 2018 & $\begin{array}{c}69.033 \\
2\end{array}$ & $\begin{array}{c}73.252 \\
3\end{array}$ & $\begin{array}{c}72.652 \\
2\end{array}$ & $\begin{array}{c}14.904 \\
6\end{array}$ & 0.346 & $94 \%$ \\
\hline 2019 & 71.033 & 77.028 & 76.875 & 10.706 & 0.337 & $99 \%$ \\
& 3 & 3 & 0 & 8 & & \\
\hline
\end{tabular}

Table 17. Problems on Initial Grades and Limit Requirement.

\begin{tabular}{|c|c|c|c|c|c|c|}
\hline \multicolumn{7}{|c|}{ PROBLEMS ON INITIAL GRADES AND LIMIT } \\
REQUIREMENT \\
\hline Year & $\begin{array}{c}\text { Shape } \\
\text { Param } \\
\text { eter }\end{array}$ & $\begin{array}{c}\text { Scala } \\
\text { Param } \\
\text { eter }\end{array}$ & Mean & $\begin{array}{c}\text { Standa } \\
\text { r } \\
\text { Deviat } \\
\text { ion }\end{array}$ & $\begin{array}{c}\text { The } \\
\text { Peak } \\
\text { value }\end{array}$ & $\begin{array}{c}\text { Surv } \\
\text { ival } \\
(70)\end{array}$ \\
\hline 2017 & $\begin{array}{c}68.823 \\
0\end{array}$ & $\begin{array}{c}72.500 \\
8\end{array}$ & $\begin{array}{c}71.080 \\
0\end{array}$ & 7.5769 & 0.349 & $88 \%$ \\
\hline 2018 & $\begin{array}{c}67.903 \\
3\end{array}$ & $\begin{array}{c}66.124 \\
4\end{array}$ & $\begin{array}{c}67.240 \\
0\end{array}$ & 7.7797 & 0.375 & $0 \%$ \\
\hline 2019 & 74.023 & 75.000 & 78.320 & 8.6348 & 0.363 & $99 \%$ \\
& 2 & 2 & 0 & & & \\
\hline
\end{tabular}

The followings are data plot presentations of stabilization value to determine the Maximum Likelihood and data distributions that have been analyzed from Table 3.1 to Table 3.17:

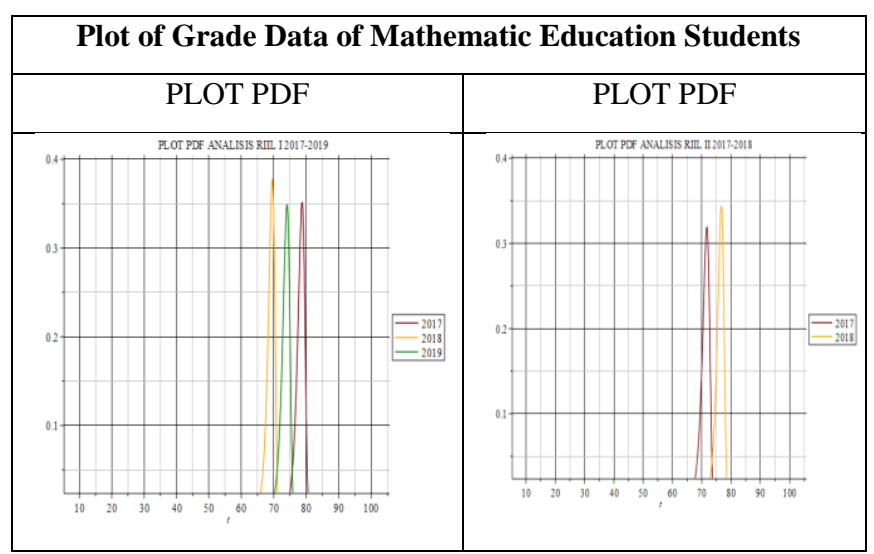

Fig. 1: PDF Plot of Real Analysis I and Real Analysis II

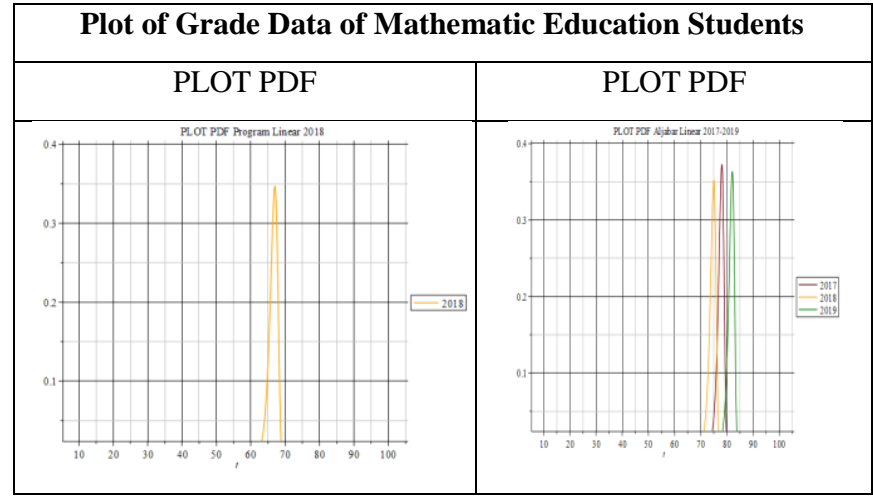

Fig. 2: PDF Plot of Linear Program and Linear Algebra

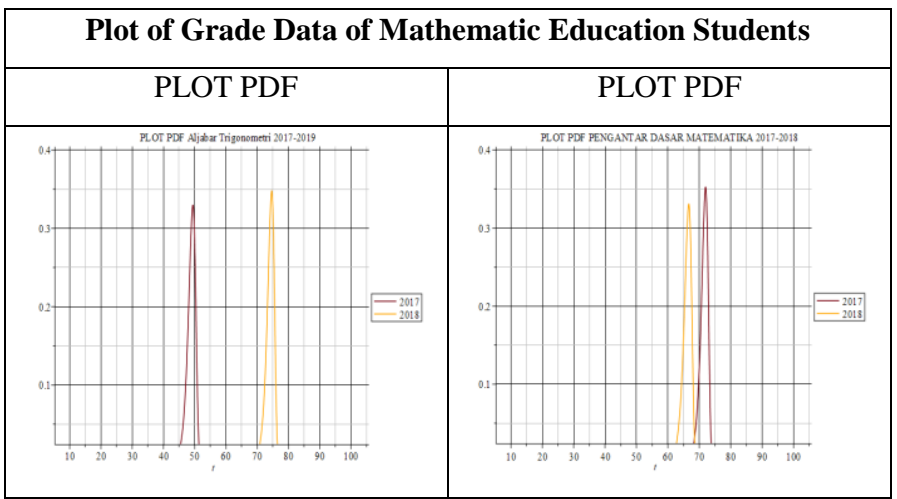

Fig. 3: PDF Plot of Trigonometric Algebra and Introduction to Basic Math

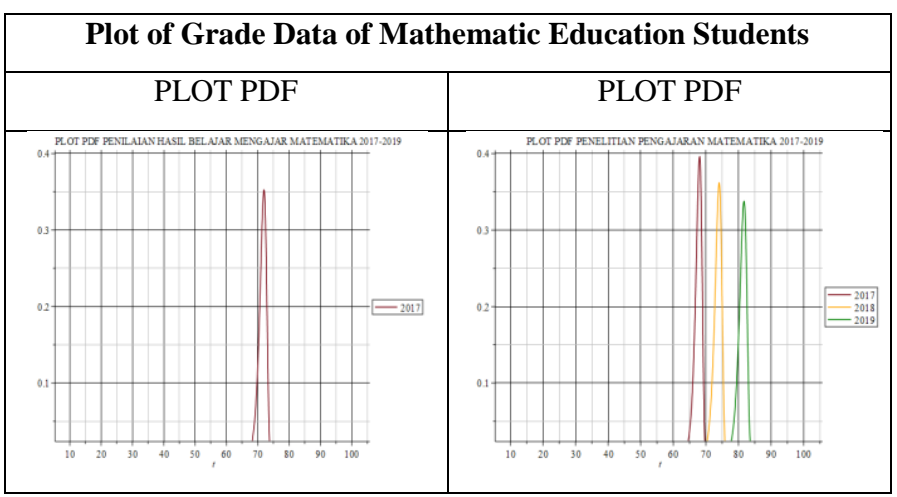

Fig. 4: PDF Plot of Evaluation on Teaching and Learning Math Achievement and Research on Math Teaching

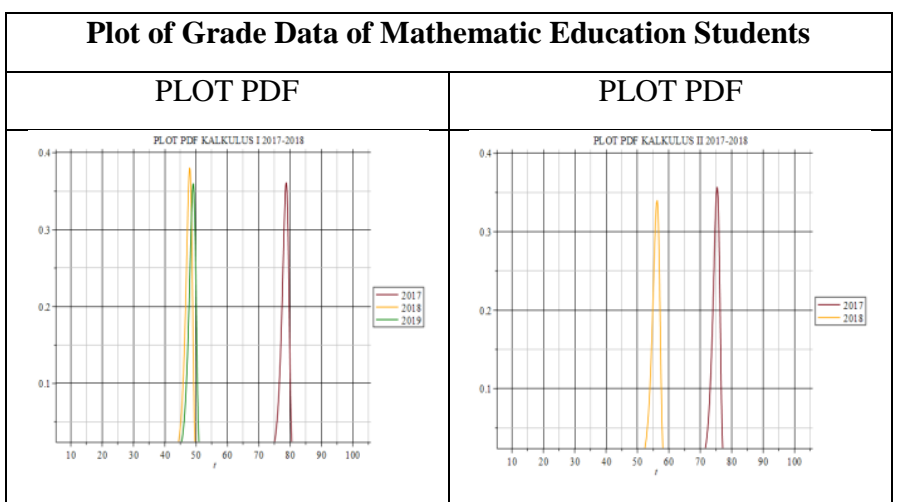

Fig. 5: PDF Plot of Calculus I and Calculus II 


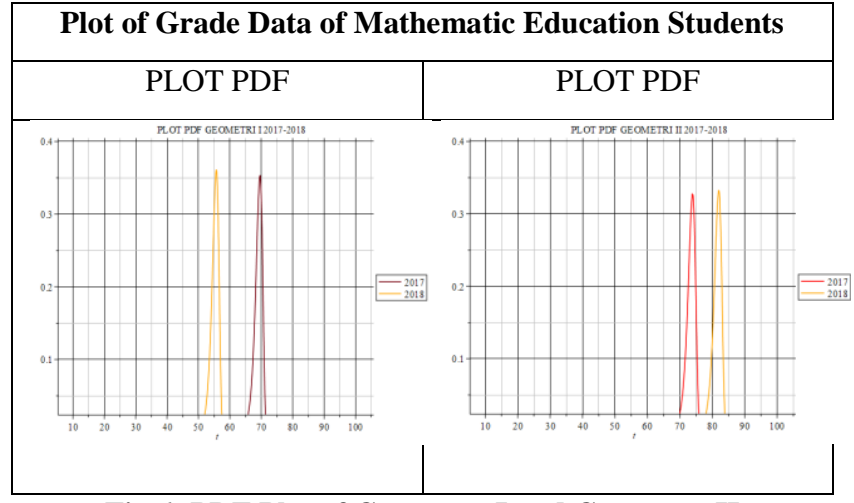

Fig 6: PDF Plot of Geometry I and Geometry II

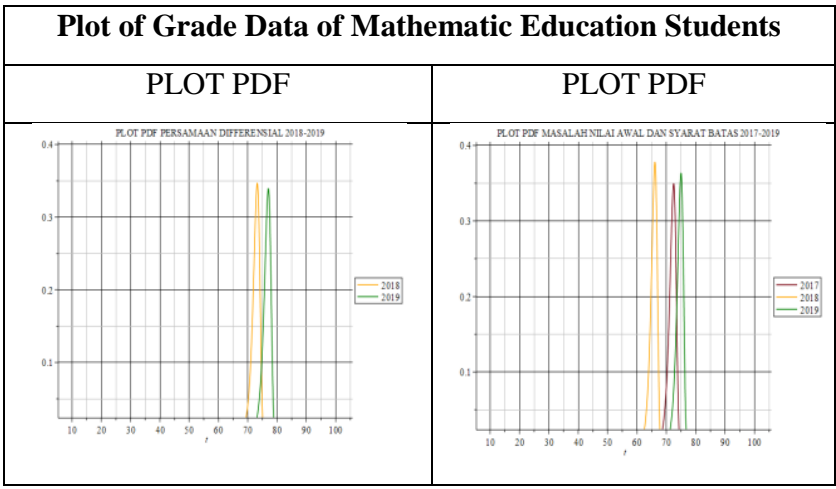

Fig. 7: PDF Plot of Differential Equation and Initial Grade Problems and Limit Requirement

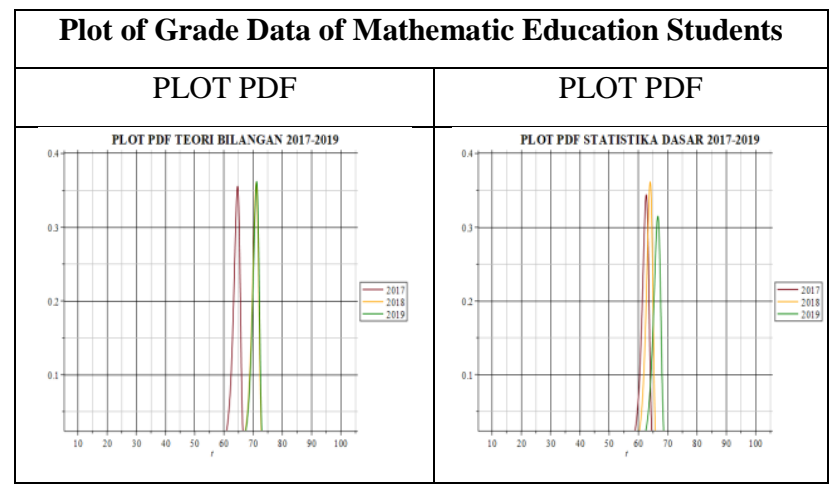

Fig. 8: PDF Plot of Number Theory and Basic Statistics

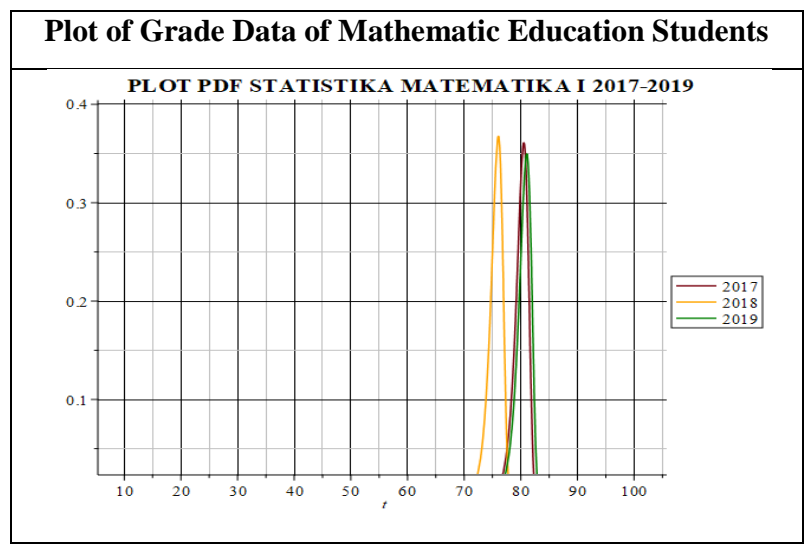

Fig. 9: PDF Plot of Math Statistics

Based on the given descriptions on the tables and figures, the learning achievements of Mathematic Education Students, Faculty of Teacher Training and Education, Cenderawasih University are categorized in Table 3.18 as follows:
Table 18. Description on the Results of 17 subjects at Mathematic Education Program

\begin{tabular}{|c|c|c|}
\hline $\mathrm{NO}$ & SUBJECTS & CATEGORY \\
\hline 1 & Real Analysis I & Good \\
\hline 2 & Real Analysis II & Sufficient \\
\hline 3 & Linear Program & Sufficient \\
\hline 4 & Linear Algebra & Good \\
\hline 5 & Trigonometric Algebra & Sufficient \\
\hline 6 & Geometry I & Less \\
\hline 7 & Geometry II & Good \\
\hline 8 & Calculus I & Less \\
\hline 9 & Calculus II & Less \\
\hline 10 & Research on Math Teaching & Good \\
\hline 11 & $\begin{array}{c}\text { Evaluation on Math Teaching and } \\
\text { Learning Achievement }\end{array}$ & Sufficient \\
\hline 12 & Differential Equation & Good \\
\hline 13 & $\begin{array}{l}\text { Problems on Initial Grades and } \\
\text { Limit Requirement }\end{array}$ & Sufficient \\
\hline 14 & Introduction to Basic Math & Sufficient \\
\hline 15 & Number Theory & Sufficient \\
\hline 16 & Basic Math Statistics & Sufficient \\
\hline 17 & Math Statistics I & Good \\
\hline
\end{tabular}

Based on the categorization of 17 subjects above, there are 6 subjects classified good. The subjects are Real Analysis I, Linear Algebra, Geometry II, Research on Math Teaching, Differential Equation and Math Statistics I. Eight subjects are categorized Sufficient namely Real Analysis II, Linear Program, Trigonometric Algebra, Evaluation on Math Teaching and Learning Achievement, Problems on Initial Grades and Limit Requirement, Introduction to Basic Math, Number Theory, and Basic Math Statistics. There are 3 subjects identified less i.e. Geometry I, Calculus I and Calculus II. To sum up in general the students' learning achievement is categorized at sufficient level. The level of reliability is also classified at adequate level $(0.2>$ $\mathrm{R}>0.4$ ). Referring to this result, it is necessary to improve the learning quality to develop the insights and knowledge of Mathematic Education. These categories of sufficient and less are calculated to have the percentage of 64 while the good category reaches $36 \%$. These percentages can be a matter of evaluation for the study program so as to work hard in improving students' learning achievement both internally and externally. This should be done in order to reach the position of good category for the all subjects. The priority of improvement is on the sufficient and less categorized subjects. Therefore lecturers are supposed to be active in facilitating, mediating and accommodating the learning process like discussion, guiding, continuously planned motivation, etc. in order to enhance teaching and learning achievement in the study program including assisting students in identifying strategies of solving problems and interpersonal communication.

\section{CONCLUSION}

Based on the results of data analysis, it can be concluded that one of important aspects in inferential statistics is to estimate a 
parameter of a population via data analysis collected from that population. A summary is made after the process of estimations from the parameters based on the sample data information of the population. Weibull distribution uses the Maximum Likelihood Estimation method with the iteration of Newton Raphson. The results present numerical and graphs descriptions and predictions properly in order to analyze and interpret the data of learning achievement of Mathematic Education Students at Cenderawasih University.

\section{ACKNOWLEDGMENTS}

Thanks to the Dean of the Teaching and Education faculty and the Head of the mathematics education study program at Cenderawasih University who supported so that this research could be completed well.

\section{REFERENCES}

[1] Khuri, A. I., 2003, Advanced Calculus WithApplications In Statistics, second Edition, John Wiley \& Sons.

[2] Agresti,A.2013. Categorical Data Analysis, Third Edition.John Wiley \& Sons:NewYork.

[3] Sudjana,Nana. 2012. Assessment of Teaching and Learning Results. Bandung : PT Remaja Rosdakarya.

[4] George,F, 2014. A Comparison of Shape and Scale
Estimators of the Two-Parameter Weibull Distribution. Journal of Modern Applied Statistical Methods,Vol 13,No.1,23-35.

[5] Majid, Abdul. 2014. Authentic Assessment Process and Learning Outcomes. Bandung: PT.Remaja Rosdakarya.

[6] Manurung,H.E.,Sediyono,E.,Sembiring,I.,2014.Implementi ng Weibull Distribution to Generate Prediction Function for National Final Examination in Papua Province. International Journal of Computer Applications, Vol.87, No.6,0975-8887.

[7] Sroyer,A.,Tandiangnga,T.,Reba,Felix.,Beno,I.S.,2018. Comparison of Parameter Estimation Methods to Determine the Frequency Data Magnitude of Aftershock in Nabire, Papua. Journal of Science and Science Education,Vol.2,No.2,63-67.

[8] Indrayani,E.,Dimara,L.,Paiki,K.,Reba,F,2018. The Analysis of Phytoplankton Abundance Using Weibull Distribution (A Case Study in the Coastal Area of East Yapen in the Regency of Yapen Islands, Papua. Journal of Education and Learning,Vol. 7, No. 3,1927-5269.

[9] Yang, W.Y., et al. 2005. Applied Numerical Methods using Matlab. John Wiley\& Sons, Inc. 